\title{
Invasive urothelial carcinoma, lymphoma- like/plasmacytoid variant, successfully treated by radical cystectomy with adjuvant chemotherapy: a case report
}

\author{
Mari Ohtaka', Takashi Kawahara ${ }^{1,2^{*}}$ (D, Yohei Kumano ${ }^{1}$, Yoko Maeda', Takuya Kondo' ${ }^{1}$, Taku Mochizuki', \\ Hiroaki Ishida', Yusuke Hattori ${ }^{1}$, Jun-ichi Teranishi ${ }^{1}$, Yasuhide Miyoshi ${ }^{1}$, Yasushi Yumura ${ }^{1}$, Masahiro Yao², \\ Yoshiaki Inayama ${ }^{3}$ and Hiroji Uemura'
}

\begin{abstract}
Background: Invasive urothelial carcinoma, lymphoma-like/plasmacytoid variant, is a rare histological type of bladder cancer similar to plasma cells and is an aggressive variant of urothelial carcinoma associated with a poor prognosis.

Case presentation: A 41-year-old Asian man was referred to our hospital due to macroscopic hematuria. Cystoscopy detected a non-papillary tumor, and a transurethral resection of the bladder tumor revealed PT1NOMO bladder cancer. A pathological examination showed high-grade invasive urothelial carcinoma and a component of signet ring cell carcinoma. A follow-up of the transurethral resection with radical cystectomy was carried out, and a pathological examination showed infiltrating urothelial carcinoma, with partial features of the plasmacytoid variant. We added chemotherapy treatment with gemcitabine and cisplatin for two cycles. Our patient has been free from recurrence for 2 years.
\end{abstract}

Conclusions: We herein report the case of a patient with a plasmacytoid variant of urothelial carcinoma controlled with radial cystectomy and subsequent chemotherapy.

Keywords: Urothelial carcinoma, Plasmacytoid variant, Gemcitabine, Cisplatin, Radical cystectomy

\section{Background}

Invasive urothelial carcinoma, lymphoma-like/plasmacytoid variant (PUC), is a rare histological type of bladder cancer similar to plasma cells and is an aggressive variant of urothelial carcinoma (UC) associated with a poor prognosis. The first report for PUC was provided by Sahin et al.; to date, only approximately 100 cases of PUC have been reported in the English literature [1-5]. We herein report a case of a patient with plasmacytoid urothelial carcinoma that

\footnotetext{
*Correspondence: takashi_tk2001@yahoo.co.jp

'Department of Urology and Renal Transplantation, Yokohama City University Medical Center, 4-57 Urafune-cho Minami-ku, Yokohama 232-0024, Japan

${ }^{2}$ Department of Urology, Yokohama City University Graduate School of Medicine, 4-57 Urafune-cho Minami-ku, Yokohama 232-0024, Japan Full list of author information is available at the end of the article
}

was successfully controlled with chemotherapy following radical cystectomy.

\section{Case presentation}

A 41-year-old Asian man was referred to our hospital due to macroscopic hematuria. His past history included middle ear cholesteatoma and he had no smoking history. Cystoscopy detected a non-papillary tumor, and a transurethral resection of the bladder tumor (TUR-Bt) and a magnetic resonance imaging (MRI) scan revealed cT3NOMO bladder cancer.

\section{Laboratory data at the time of admission}

The hematological and biochemical data showed no abnormal findings. A urinary analysis resulted in the following: pH 6.5, red blood cell count 10-19/high-power field (HPF), white blood cell count 1-4/HPF, protein 


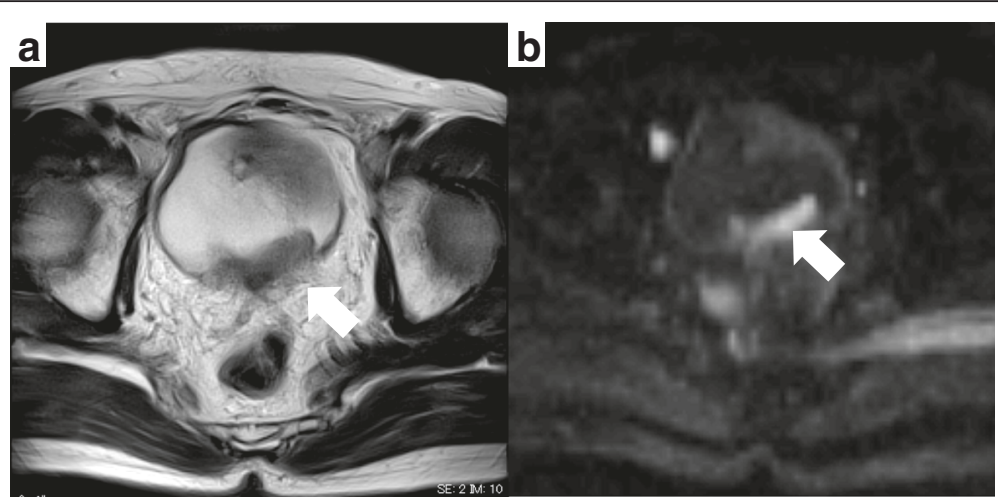

Fig. 1 Magnetic resonance imaging findings in (a) T2-weighted and (b) diffusion-weighted images. The mass was believed to be invasive outside of the bladder wall (arrow)

negative, and glucose negative. The urinary cytology was class III.

\section{Imaging findings}

An MRI scan showed that the tumor extended over the bladder, as seen in Fig. 1. No obvious distal and local lymphadenopathy was observed on a non-contrast computed tomography (CT) scan.

\section{Operative procedure}

Our patient underwent a TUR-Bt, and a pathological examination revealed urothelial carcinoma (high-grade, pT1, G2) and signet ring cell carcinoma was found in a portion of the bladder tumor. Two months after the TUR-Bt, a radical cystectomy was performed according to the tumor grade of malignancy and the imaging findings (Fig. 2).

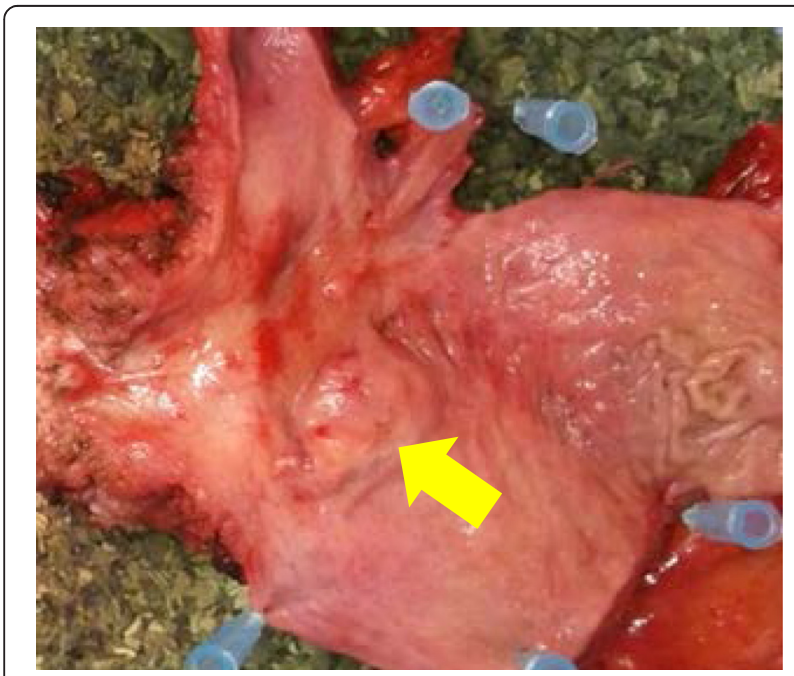

Fig. 2 A surgical specimen from the radical cystectomy. The tumor was located in the bladder trigone (arrow)

\section{Pathological findings}

Grossly, the infiltrating neoplasm, measuring $25 \times 20 \mathrm{~mm}$ in size, was located in the bladder trigone. The pathological stage was pT2bN0M0. Histologically, the neoplasm was composed of isolated atypical cells that had relatively abundant eosinophilic cytoplasm with eccentric nuclei, showing moderate pleomorphism. Perinuclear pale regions were occasionally seen. These features partly resembled plasma cells (Fig. 3). The immunohistochemical profile of the tumor cells was positive for keratin CAM5.2 and CK20, but negative for CK7, CD56, chromogranin A, synaptophysin, CD20, CD79a, kappa and lambda. Thus, the diagnosis of infiltrating high-grade urothelial carcinoma, plasmacytoid variant, was made. Plasma cells have a similar form to signet ring cells, thus it is difficult to distinguish these cells. However, plasma cells frequently show CD138 positivity, which may be useful for discriminating between the two types of cells.

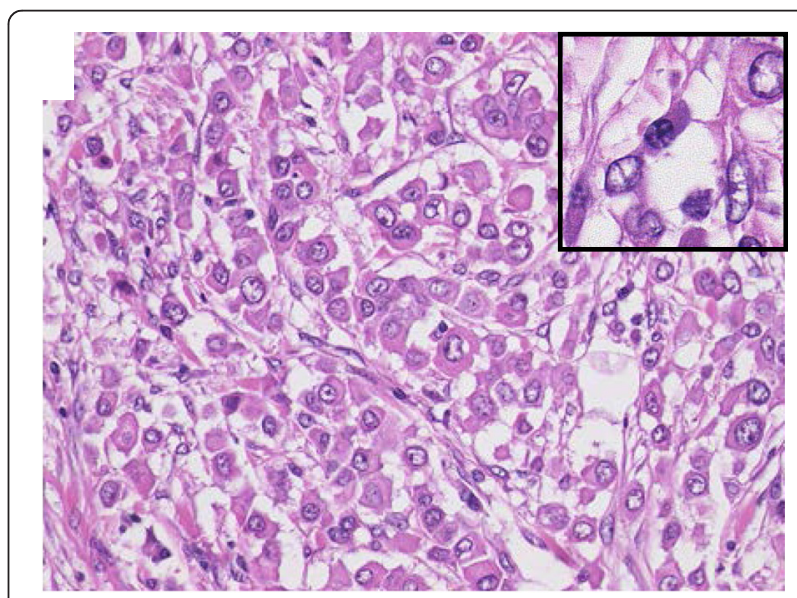

Fig. 3 Hematoxylin and eosin stain. Plasmacytoid urothelial carcinoma was characterized by isolated cells with uneven nuclear distribution. Invasive plasma cell was founded sparsely (enlarged part of image) 


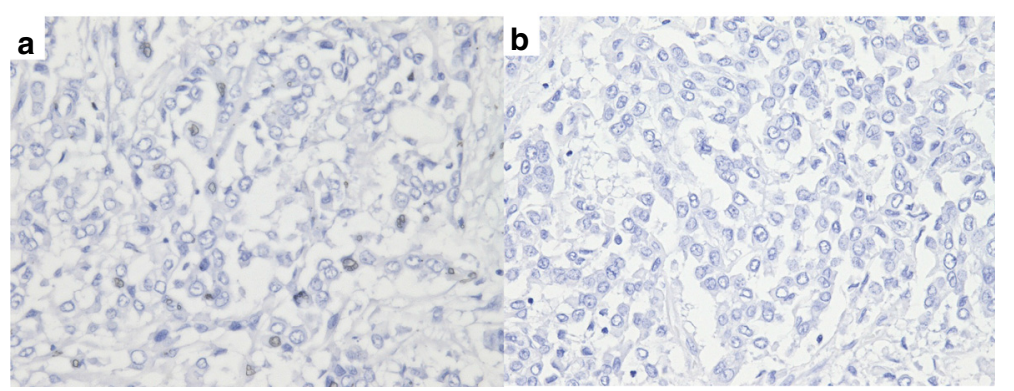

Fig. 4 Immunohistochemistry for PD-1 (a) and PD-L1 (b) expression. There was no positive expression in both PD-1 and PD-L1

\section{Immunohistochemistry for PD-1 and PD-L1}

An immunohistochemical analysis was performed on 5 - $\mu$ m-thick sections. The slides were dewaxed with xylene and hydrated with gradient ethanol, and microwaved at the high level for 2 minutes and then at the medium-low level for 30 minutes for heat antigen retrieval (Target Retrieval Solution pH 9, Dako, Carpinteria, CA, USA). After $3 \%$ hydrogen peroxidase blocking, the samples were incubated overnight at $4{ }^{\circ} \mathrm{C}$ with a primary antibody to PD-1 (dilution 1:50, Santa Cruz Biotechnology, Santa Cruz, CA, USA) or PD-L1 (dilution 1:50, Santa Cruz Biotechnology). The slides were then treated with a broadspectrum secondary antibody (Invitrogen, Grand Island, NY, USA) and washed (Envision FLEX Wash Buffer, Dako). After diaminobenzidine staining, the slides were counterstained with hematoxylin, dehydrated with gradient ethanol and xylene, and then sealed. There were no positive finding (Fig. 4).

\section{Postoperative course}

Two months after the total cystectomy, adjuvant chemotherapy with gemcitabine and cisplatin was administered (G: $2,000 \mathrm{mg} / \mathrm{m}^{2}, \mathrm{C}: 63 \mathrm{mg} / \mathrm{m}^{2}$ ). We used the following dosing schedule: day $1 \mathrm{G}: 1,000 \mathrm{mg} / \mathrm{m}^{2}$, day $2 \mathrm{C}: 63 \mathrm{mg} / \mathrm{m}^{2}$, and day $8 \mathrm{G}: 1,000 \mathrm{mg} / \mathrm{m}^{2}$ that was administered in two cycles. No adverse event was observed. We followed up our patient with cytology and a CT scan every 3 months. Our patient has not experienced any recurrence of bladder cancer for 2 years following the radical cystectomy.

\section{Discussion}

PUC is a rare histological type of bladder cancer similar to plasma cells and is an aggressive variant of UC associated with a poor prognosis [6]. The first case of PUC was reported by Sahin et al.; to date, only approximately100 cases of PUC have been reported in the English literature $[1-3,5]$. Most patients were in their $60 \mathrm{~s}$; the male-to-female ratio was 9:1. This variant is typically diagnosed at an advanced pathological stage (64 \% pT3, $23 \%$ pT4), showing metastases in $60 \%$ of the patients [3]. This tumor initially presents as a high- grade, high-stage lesion, and diffusely invades the bladder wall; however, patients have no specific symptoms, which can lead to a delayed diagnosis and poor prognosis. Furthermore, PUC is difficult to differentiate from signet ring cell carcinoma of the urinary bladder due to overlap in the clinical and morphological presentation [4]. In the present case, the initial diagnosis was signet ring cell carcinoma, thus we performed a radical cystectomy because of the poor prognosis. However, the subsequent pathological examination revealed infiltrating high-grade urothelial carcinoma with partial features of the plasmacytoid variant, thus we administered chemotherapy.

Most urological guidelines recommend adjuvant cisplatin-based chemotherapy as the therapy of choice in locally advanced bladder cancer. However, it has been reported that tumors with variant histology are associated with a higher risk of progression than conventional highgrade UC. Keck et al. reported that patients suffering from PUC have the worst clinical outcome regarding overall survival compared to conventional UC [7]. We searched for other cases of PUC that were treated with cystectomy and chemotherapy. Almost all cases were treated with cystectomy and adjuvant chemotherapy. All cases were at an advanced stage, and the chemotherapy consisted of methotrexate, vinblastine, doxorubicin and cisplatin (M-

Table 1 The cases of PUC that were treated with cystectomy and adjuvant chemotherapy

\begin{tabular}{|c|c|c|c|c|}
\hline First author & TNM stage & Chemotherapy & Outcome & OS \\
\hline Kohno [12] & T4NOMO & $M-V A C \times 2$ & Survival & $18 \mathrm{~m}$ \\
\hline Kawashima [13] & T3aNOMO & $M-V A C \times 2$ & Survival & $11 \mathrm{~m}$ \\
\hline \multirow[t]{2}{*}{ Fritsche [14] } & T4bN2M0 & $\mathrm{GC} \times 4$ & Survival & $16 \mathrm{~m}$ \\
\hline & T4aNOMO & $\mathrm{GC} \times 5$ & Death & $29 \mathrm{~m}$ \\
\hline Soylu [15] & $\mathrm{T} 2 \mathrm{bNxM0}$ & 5FU+leucovorin & Death & $18 \mathrm{~m}$ \\
\hline Aldousari [16] & T3bNOMO & GC & Death & $6 \mathrm{~m}$ \\
\hline Hayashi [17] & T4bNOMO & $\mathrm{GC} \times 2$ & Death & $9 \mathrm{~m}$ \\
\hline Present case & T2bNOMO & $\mathrm{GC} \times 2$ & Survival & $26 \mathrm{~m}$ \\
\hline
\end{tabular}

PUC invasive urothelial carcinoma, lymphoma-like/plasmacytoid variant, TNM tumornode-metastasis, OS overall survival, $M-V A C$ methotrexate, vinblastine, doxorubicin and cisplatin, GC gemcitabine and cisplatin, 5FU 5-fluorouracil, $m$ months 
VAC) or GC. Cystectomy and adjuvant chemotherapy with GC was relatively effective for the management of PUC in terms of overall survival (Table 1). Furthermore, Kaimakliotis et al. reported that the management of PUC should be aggressive and that cystectomy should be performed at all stages [8]. They also reported that although it is unclear whether PUC is independently associated with a poor prognosis, the prognosis in PUC is poor due to the higher stage at the time of diagnosis [9].

Recently, an anti-PD-L1 drug was found to have a rapid and ongoing response in patients with urothelial carcinoma in a phase 1 study. Interestingly, this drug showed higher efficacy in patients whose tumor-infiltrating cells showed high levels of PD-L1 expression [10]. Boorjian et al. reported that higher PD-L1 expression in tumor cells was associated with the presence of advanced disease in patients with urothelial carcinoma and that it was correlated with a poor prognosis after radical cystectomy [11]. In our case, the tumor did not express PD-1 or PD-L1.

Although we predicted a poor prognosis for this variant, the tumor was successfully treated with a combination of radical cystectomy and adjuvant chemotherapy, and our patient has remained free of any sign of recurrence of bladder cancer for 2 years after the operation.

\section{Conclusions}

We herein described the case of a patient with PUC controlled with chemotherapy following radical cystectomy.

\section{Consent}

Written informed consent was obtained from the patient for publication of this case report and accompanying images. A copy of the written consent is available for review by the Editor-in-Chief of this journal.

\section{Abbreviations}

CT: Computed tomography; GC: Gemcitabine and cisplatin; HPF: high-power field; MRI: Magnetic resonance imaging; M-VAC: Methotrexate, vinblastine, doxorubicin and cisplatin; PUC: Invasive urothelial carcinoma, lymphoma-like/ plasmacytoid variant; TUR-Bt: Transurethral resection of a bladder tumor; UC: Urothelial carcinoma.

\section{Competing interests}

The authors declare that they have no competing interests.

\section{Authors' contributions}

$M O, T M, H I, Y H, J T$, and YMa performed the operation and YK, YM, TKo, YH, and YY conducted the patient follow-up. YI performed the pathological diagnosis. MO and TKa conceived of the study, participated in its design and wrote the manuscript. YMi and $\mathrm{HU}$ helped to check the draft. All authors read and approved the final manuscript.

\section{Author details}

'Department of Urology and Renal Transplantation, Yokohama City University Medical Center, 4-57 Urafune-cho Minami-ku, Yokohama 232-0024, Japan. ${ }^{2}$ Department of Urology, Yokohama City University Graduate School of Medicine, 4-57 Urafune-cho Minami-ku, Yokohama 232-0024, Japan. ${ }^{3}$ Department of Diagnostic Pathology, Yokohama City University Medical Center, 4-57 Urafune-cho Minami-ku, Yokohama 232-0024, Japan.

Received: 25 October 2015 Accepted: 6 January 2016

Published online: 08 March 2016

\section{References}

1. Wang Z, Lu T, Du L, Hu Z, Zhuang Q, Li Y, et al. Plasmacytoid urothelial carcinoma of the urinary bladder: a clinical pathological study and literature review. Int J Clin Exp Pathol. 2012:5:601-8.

2. Keck B, Stoehr R, Wach S, Rogler A, Hofstaedter F, Lehmann J, et al. The plasmacytoid carcinoma of the bladder-rare variant of aggressive urothelial carcinoma. Int J Cancer. 2011;129:346-54.

3. Gonzalez-Roibon ND, Chaux A, Al-Hussain T, Osunkoya AO, Bezerra SM, Hicks J, et al. Dysregulation of mammalian target of rapamycin pathway in plasmacytoid variant of urothelial carcinoma of the urinary bladder. Hum Pathol. 2013:44:612-22.

4. Qin M, Wang G, Sun Y, He Q. Plasmacytoid urothelial carcinoma of the bladder. Indian J Pathol Microbiol. 2014;57:320-2.

5. Sahin AA, Myhre M, Ro JY, Sneige N, Dekmezian RH, Ayala AG. Plasmacytoid transitional cell carcinoma. Report of a case with initial presentation mimicking multiple myeloma. Acta Cytol. 1991;35:277-80.

6. Kawahara T, Oshiro H, Sekiguchi Z, Ito H, Makiyama K, Uemura H, et al. High-grade invasive urothelial carcinoma with focal plasmacytoid differentiation successfully treated by transurethral resection followed by chemoradiotherapy. Int J Urol. 2011;18:851-3.

7. Keck B, Wach S, Stoehr R, Kunath F, Bertz S, Lehmann J, et al. Plasmacytoid variant of bladder cancer defines patients with poor prognosis if treated with cystectomy and adjuvant cisplatin-based chemotherapy. BMC Cancer. 2013;13:71.

8. Kaimakliotis HZ, Monn MF, Cary KC, Pedrosa JA, Rice K, Masterson TA, et al. Plasmacytoid variant urothelial bladder cancer: is it time to update the treatment paradigm? Urol Oncol. 2014;32:833-8.

9. Kaimakliotis HZ, Monn MF, Cheng L, Masterson TA, Cary KC, Pedrosa JA, et al. Plasmacytoid bladder cancer: variant histology with aggressive behavior and a new mode of invasion along fascial planes. Urology. 2014;83:1112-6.

10. Brower V. Anti-PD-L1 antibody active in metastatic bladder cancer. Lancet Oncol. 2015;16:e11.

11. Boorjian SA, Sheinin Y, Crispen PL, Farmer SA, Lohse CM, Kuntz SM, et al. T-cell coregulatory molecule expression in urothelial cell carcinoma: clinicopathologic correlations and association with survival. Clin Cancer Res. 2008;14:4800-8.

12. Kohno T, Kitamura M, Akai H, Takaha M, Kawahara K, Oka T: Plasmacytoid urothelial carcinoma of the bladder. Int J Urol 2006, 13(4):485-486.

13. Kawashima A, Ujike T, Nin M, Nishimura K, Miyoshi S: [Plasmacytoid urothelial carcinoma of the bladder: a case report]. Nihon Hinyokika Gakkai Zasshi 2009, 100(5):590-594.

14. Fritsche HM, Burger M, Denzinger S, Legal W, Goebell PJ, Hartmann A: Plasmacytoid urothelial carcinoma of the bladder: histological and clinical features of 5 cases. J Urol 2008, 180(5):1923-1927.

15. Soylu A, Aydin NE, Yilmaz U, Kutlu R, Gunes A: Urothelial carcinoma featuring lipid cell and plasmacytoid morphology with poor prognostic outcome. Urology 2005, 65(4):797.

16. Aldousari S, Sircar K, Kassouf W: Plasmacytoid urothelial carcinoma of the bladder: a case report. Cases J 2009, 2:6647.

17. Hayashi T, Tanigawa G, Fujita K, Imamura R, Nakazawa S, Yamamoto Y, Hosomi M, Shimazu K, Fushimi H, Yamaguchi S: Two cases of plasmacytoid variant of urothelial carcinoma of urinary bladder: systemic chemotherapy might be of benefit. Int J Clin Oncol 2011, 16(6):759-762.

\section{Acknowledgements}

Rie Shimizu supported the immunohistochemical study. Grants from the Uehara Memorial Foundation, the Tokyo Biochemical Research Foundation, the Japanese Foundation for Research and Promotion of Endoscopy, and an International Exchange Grant from Kato Memorial Bioscience Foundation were provided to TK. There are no applicable grant numbers. 